doi: $10.2306 /$ scienceasia1513-1874.2012.38.408

\title{
An alternative Jensen's functional equation on semigroups
}

\author{
Paisan Nakmahachalasint \\ Department of Mathematics and Computer Science, Faculty of Science, Chulalongkorn University, \\ Bangkok 10330 Thailand
}

e-mail: Paisan.N@chula.ac.th

Received 22 Aug 2012

Accepted 30 Oct 2012

ABSTRACT: We study the alternative Jensen's functional equation $f(x) \pm 2 f(x y)+f\left(x y^{2}\right)=0$ when $f$ is a function from a semigroup or a group to a uniquely divisible abelian group.

KEYWORDS: Cauchy functional equation, additive function, functional equation on groups

\section{INTRODUCTION}

The alternative Cauchy functional equation

$$
\|f(x+y)\|=\|f(x)+f(y)\|
$$

has been studied by a number of authors (e.g., Kuczma ${ }^{1,2}$ ). Forti ${ }^{3}$ solved a more general problem of the form

$$
\begin{aligned}
&\{c f(x+y)-a f(x)-b f(y)-d\} \\
& \times\{f(x+y)-f(x)-f(y)\}=0
\end{aligned}
$$

by extending the work of $\mathrm{Ger}^{4}$ as well as that of Forti and Paganoni ${ }^{5}$ as mentioned in the paper by Paganoni and Rätz ${ }^{6}$. Inspired by the work on alternative Cauchy functional equations, we will generalize Jensen's functional equation

$$
f\left(\frac{x+y}{2}\right)=\frac{f(x)+f(y)}{2}
$$

to an alternative version

$$
\left\|f\left(\frac{x+y}{2}\right)\right\|=\left\|\frac{f(x)+f(y)}{2}\right\| .
$$

In order to study functions $f: S \rightarrow G^{*}$, where $(S, \cdot)$ is a semigroup and $\left(G^{*},+\right)$ is a group, it would be appropriate to state Jensen's functional equation as

$$
f(x)-2 f(x y)+f\left(x y^{2}\right)=0,
$$

and propose the alternative Jensen's functional equation

$$
f(x) \pm 2 f(x y)+f\left(x y^{2}\right)=0 .
$$

Jensen's functional equation $f(x y)+f\left(x y^{-1}\right)=$ $2 f(x)$ on groups has been studied by several authors (e.g., $\mathrm{Ng}^{7}$ and Parnami $^{8}$ ), but the alternative version (3) has not yet been investigated.

In this paper, we will develop a theorem concerning (3) on semigroups and will give illustrating examples on some specific semigroups. In addition, we will extend the result to functions defined on groups and will eventually show that in the case of functions $f$ from a 2-divisible group to a uniquely divisible abelian group, the alternative Jensen's functional equation (3) is equivalent to Jensen's functional equation (2).

\section{AUXILIARY LEMMAS}

In this and the next section, we shall let $(S, \cdot)$ be a semigroup and let $\left(G^{*},+\right)$ be a uniquely divisible abelian group. We denote the set of all positive integers by $\mathbb{N}$ and the set of all integers by $\mathbb{Z}$.

Throughout this section, there will be many substitutions in the alternative Jensen's functional equation (3), and it will be convenient to introduce the following notation. For a function $f: S \rightarrow G^{*}$, denote the functionals $J_{f}^{+}, J_{f}^{-}: S^{2} \rightarrow G^{*}$ by

$$
\text { and } \begin{aligned}
J_{f}^{+}(x, y) & =f(x)+2 f(x y)+f\left(x y^{2}\right) \\
J_{f}^{-}(x, y) & =f(x)-2 f(x y)+f\left(x y^{2}\right) .
\end{aligned}
$$

It is worth noting that Jensen's functional equation (2) is equivalent to $J_{f}^{-}(x, y)=0$. We shall denote the statement

$\mathcal{P}_{f}(x, y)=\left(\left(J_{f}^{+}(x, y)=0\right) \quad\right.$ or $\left.\quad\left(J_{f}^{-}(x, y)=0\right)\right)$.

The set of all solutions of (3) will be denoted by

$$
\mathcal{A}(S)=\left\{f: S \rightarrow G^{*} \mid \mathcal{P}_{f}(x, y) \text { for all } x, y \in S\right\}
$$


We will now prove four lemmas constituting the theorem in the next section when the alternatives in $\mathcal{P}_{f}(x, y)$ and $\mathcal{P}_{f}(x y, y)$ are decided.

Lemma 1 Let $f \in \mathcal{A}(S)$. For $x, y \in S$, if $J_{f}^{-}(x, y)=0$ and $J_{f}^{-}(x y, y)=0$, then $J_{f}^{-}\left(x y^{2}, y\right)=$ 0 .

Proof: Suppose there exist $a, b \in S$ such that $J_{f}^{-}(a, b)=0$ and $J_{f}^{-}(a b, b)=0$, but $J_{f}^{-}\left(a b^{2}, b\right) \neq 0$; that is, $J_{f}^{+}\left(a b^{2}, b\right)=0$. Thus

$$
J_{f}^{-}(a, b)+2 J_{f}^{-}(a b, b)-J_{f}^{+}\left(a b^{2}, b\right)=0,
$$

which simplifies to

$$
f(a)-4 f\left(a b^{2}\right)-f\left(a b^{4}\right)=0 .
$$

Let $k=f\left(a b^{2}\right)$ and consider the two cases on $\mathcal{P}_{f}\left(a, b^{2}\right)$.

(i) If $J_{f}^{+}\left(a, b^{2}\right)=0$, that is, $f(a)+2 f\left(a b^{2}\right)+$ $f\left(a b^{4}\right)=0$, then solving it with (4) gives $f(a)=$ $k$ and $f\left(a b^{4}\right)=-3 k$. From $J_{f}^{-}(a, b)=0$ and $J_{f}^{+}\left(a b^{2}, b\right)=0$, we can calculate $f(a b)=$ $f\left(a b^{3}\right)=k$.

From $\mathcal{P}_{f}\left(a b^{3}, b\right)$, we must have $f\left(a b^{5}\right) \in$ $\{5 k,-7 k\}$. Taking into account $\mathcal{P}_{f}\left(a b, b^{2}\right)$, we will get $k=0$. Hence $f\left(a b^{3}\right)=0$.

(ii) If $J_{f}^{-}\left(a, b^{2}\right)=0$, that is, $f(a)-2 f\left(a b^{2}\right)+$ $f\left(a b^{4}\right)=0$, then solving it with (4) gives $f\left(a b^{4}\right)=-k$. From $J_{f}^{+}\left(a b^{2}, b\right)=0$, we calculate $f\left(a b^{3}\right)=0$.

From both cases above, we conclude that $f\left(a b^{3}\right)=0$, which implies $J_{f}^{-}\left(a b^{2}, b\right)=0$, a contradiction.

Corollary 1 Let $f \in \mathcal{A}(S)$. For $x, y \in S$, if $J_{f}^{-}(x, y)=0$ and $J_{f}^{-}(x y, y)=0$, then $J_{f}^{-}\left(x y^{n}, y\right)=$ 0 and $f\left(x y^{n}\right)=f(x)+n(f(x y)-f(x))$ for all $n \in \mathbb{N}$.

Proof: Repeatedly apply Lemma 1 to get $J_{f}^{-}\left(x y^{n}, y\right)=0$, which can be rearranged as

$$
f\left(x y^{n+2}\right)-f\left(x y^{n+1}\right)=f\left(x y^{n+1}\right)-f\left(x y^{n}\right) .
$$

The desired result will follow.

Lemma 2 Let $f \in \mathcal{A}(S)$. For $x, y \in S$, if $J_{f}^{+}(x, y)=$ 0 and $J_{f}^{+}(x y, y)=0$, then $J_{f}^{+}\left(x y^{2}, y\right)=0$.

Proof: Suppose there exist $a, b \in S$ such that $J_{f}^{+}(a, b)=0$ and $J_{f}^{+}(a b, b)=0$, but $J_{f}^{+}\left(a b^{2}, b\right) \neq 0$; that is, $J_{f}^{-}\left(a b^{2}, b\right)=0$. Therefore,

$$
J_{f}^{+}(a, b)-2 J_{f}^{+}(a b, b)-J_{f}^{-}\left(a b^{2}, b\right)=0,
$$

which simplifies to

$$
f(a)-4 f\left(a b^{2}\right)-f\left(a b^{4}\right)=0 .
$$

Let $k=f\left(a b^{2}\right)$ and consider the two cases on $\mathcal{P}_{f}\left(a, b^{2}\right)$.

(i) If $J_{f}^{+}\left(a, b^{2}\right)=0$, that is, $f(a)+2 f\left(a b^{2}\right)+$ $f\left(a b^{4}\right)=0$, then solving it with (5) gives $f(a)=$ $k$ and $f\left(a b^{4}\right)=-3 k$. From $J_{f}^{+}(a, b)=0$ and $J_{f}^{-}\left(a b^{2}, b\right)=0$, we can calculate $f(a b)=$ $f\left(a b^{3}\right)=-k$.

From $\mathcal{P}_{f}\left(a b^{3}, b\right)$, we compute $f\left(a b^{5}\right) \in$ $\{-5 k, 7 k\}$. From $\mathcal{P}_{f}\left(a b, b^{2}\right)$, we conclude that $k=0$. Hence $f\left(a b^{3}\right)=0$.

(ii) If $J_{f}^{-}\left(a, b^{2}\right)=0$, that is, $f(a)-2 f\left(a b^{2}\right)+$ $f\left(a b^{4}\right)=0$, then solving it with(5) gives $f(a)=$ $3 k$ and $f\left(a b^{4}\right)=-k$. From $J_{f}^{-}\left(a b^{2}, b\right)=0$, we calculate $f\left(a b^{3}\right)=0$.

From both cases above, we conclude that $f\left(a b^{3}\right)=0$, which implies $J_{f}^{+}\left(a b^{2}, b\right)=0$, a contradiction.

Corollary 2 Let $f \in \mathcal{A}(S)$. For $x, y \in S$, if $J_{f}^{+}(x, y)=0$ and $J_{f}^{+}(x y, y)=0$, then $J_{f}^{+}\left(x y^{n}, y\right)=$ 0 and $f\left(x y^{n}\right)=(-1)^{n}(f(x)-n(f(x)+f(x y))$ for all $n \in \mathbb{N}$.

Proof: Repeatedly apply Lemma 2 to get $J_{f}^{+}\left(x y^{n}, y\right)=0$, which can be rearranged as

$f\left(x y^{n+2}\right)+f\left(x y^{n+1}\right)=(-1)\left(f\left(x y^{n+1}\right)+f\left(x y^{n}\right)\right)$.

The desired result will follow.

Lemma 3 Let $f \in \mathcal{A}(S)$. For $x, y \in S$, if $J_{f}^{-}(x, y) \neq 0$ and $J_{f}^{+}(x y, y) \neq 0$, then $f\left(x y^{n}\right)=$ $-f(x) / 3$ for all $n \in \mathbb{N}$.

Proof: Suppose that $J_{f}^{-}(x, y) \neq 0$ and $J_{f}^{+}(x y, y) \neq$ 0 ; that is, $J_{f}^{+}(x, y)=0$ and $J_{f}^{-}(x y, y)=0$. Let $k=$ $f\left(x y^{2}\right)$ and consider the two cases on $\mathcal{P}_{f}\left(x y^{2}, y\right)$.

(i) If $J_{f}^{+}\left(x y^{2}, y\right)=0$, then consider

$$
J_{f}^{+}(x, y)-2 J_{f}^{-}(x y, y)+J_{f}^{+}\left(x y^{2}, y\right)=0,
$$

which simplifies to

$$
f(x)+6 f\left(x y^{2}\right)+f\left(x y^{4}\right)=0 .
$$

Comparing the above equation with $\mathcal{P}_{f}\left(x, y^{2}\right)$, we infer that $f\left(x y^{2}\right)=0$ which yields $J_{f}^{+}(x y, y)=0$, a contradiction.

(ii) If $J_{f}^{-}\left(x y^{2}, y\right)=0$, then consider

$$
J_{f}^{+}(x, y)-2 J_{f}^{-}(x y, y)-J_{f}^{-}\left(x y^{2}, y\right)=0,
$$


which simplifies to

$$
f(x)+4 f\left(x y^{2}\right)-f\left(x y^{4}\right)=0 .
$$

Consider further cases on $\mathcal{P}_{f}\left(x, y^{2}\right)$.

(a) If $J_{f}^{+}\left(x, y^{2}\right)=0$, that is, $f(x)+2 f\left(x y^{2}\right)+$ $f\left(x y^{4}\right)=0$, then solving it with (6) gives $f(x)=-3 k$ and $f\left(x y^{4}\right)=k$. From $J_{f}^{+}(x, y)=0$ and $J_{f}^{-}\left(x y^{2}, y\right)=0$, we calculate $f(x y)=f\left(x y^{3}\right)=k$. Hence we have $J_{f}^{-}(x y, y)=0$ and $J_{f}^{-}\left(x y^{2}, y\right)=0$. Now Corollary 1 gives $J_{f}^{-}\left(x y^{n}, y\right)=0$ for all $n \in \mathbb{N}$, which permits us to calculate $f\left(x y^{n}\right)=k=-f(x) / 3$ for all $n \in \mathbb{N}$.

(b) If $J_{f}^{-}\left(x, y^{2}\right)=0$, that is, $f(x)-2 f\left(x y^{2}\right)+$ $f\left(x y^{4}\right)=0$, then solving it with (6) gives $f(x)=-k$. From $J_{f}^{+}(x, y)=0$, we will have $f(x y)=0$, which leads to $J_{f}^{-}(x, y)=$ 0 , a contradiction.

Lemma 4 Let $f \in \mathcal{A}(S)$. For $x, y \in S$, if $J_{f}^{+}(x, y) \neq 0$ and $J_{f}^{-}(x y, y) \neq 0$, then $f\left(x y^{n}\right)=$ $(-1)^{n+1} f(x) / 3$ for all $n \in \mathbb{N}$.

Proof: Suppose that $J_{f}^{+}(x, y) \neq 0$ and $J_{f}^{-}(x y, y) \neq$ 0 ; that is, $J_{f}^{-}(x, y)=0$ and $J_{f}^{+}(x y, y)=0$. Let $k=$ $f\left(x y^{2}\right)$ and consider the two cases on $\mathcal{P}_{f}\left(x y^{2}, y\right)$.

(i) If $J_{f}^{+}\left(x y^{2}, y\right)=0$, then consider

$$
J_{f}^{-}(x, y)+2 J_{f}^{+}(x y, y)-J_{f}^{+}\left(x y^{2}, y\right)=0,
$$

which simplifies to

$$
f(x)+4 f\left(x y^{2}\right)-f\left(x y^{4}\right)=0 .
$$

Consider further cases on $\mathcal{P}_{f}\left(x, y^{2}\right)$.

(a) If $J_{f}^{+}\left(x, y^{2}\right)=0$, that is, $f(x)+2 f\left(x y^{2}\right)+$ $f\left(x y^{4}\right)=0$, then solving it with (7) gives $f(x)=-3 k$ and $f\left(x y^{4}\right)=k$. From $J_{f}^{-}(x, y)=0$ and $J_{f}^{+}\left(x y^{2}, y\right)=0$, we calculate $f(x y)=f\left(x y^{3}\right)=-k$. Hence we have $J_{f}^{+}(x y, y)=0$ and $J_{f}^{+}\left(x y^{2}, y\right)=0$. Now Corollary 2 gives $J_{f}^{+}\left(x y^{n}, y\right)=0$ for all $n \in \mathbb{N}$, which permits us to calculate $f\left(x y^{n}\right)=(-1)^{n} k=(-1)^{n+1} f(x) / 3$ for all $n \in \mathbb{N}$.

(b) If $J_{f}^{-}\left(x, y^{2}\right)=0$, that is, $f(x)-2 f\left(x y^{2}\right)+$ $f\left(x y^{4}\right)=0$, then solving it with (7) gives $f(x)=-k$. From $J_{f}^{-}(x, y)=0$, we will have $f(x y)=0$, which leads to $J_{f}^{+}(x, y)=$ 0 , a contradiction. (ii) If $J_{f}^{-}\left(x y^{2}, y\right)=0$, then consider

$$
J_{f}^{-}(x, y)+2 J_{f}^{+}(x y, y)+J_{f}^{-}\left(x y^{2}, y\right)=0,
$$

which simplifies to

$$
f(x)+6 f\left(x y^{2}\right)+f\left(x y^{4}\right)=0 .
$$

Comparing the above equation with $\mathcal{P}_{f}\left(x, y^{2}\right)$, we infer that $f\left(x y^{2}\right)=0$ which gives $J_{f}^{-}(x y, y)=0$, a contradiction.

\section{MAIN THEOREM AND SOME EXAMPLES}

We are now ready to establish a theorem that pieces together all the lemmas in the previous section. The theorem will serve as a foundation for solving the alternative Jensen's functional equation (3) in the examples provided thereafter. Please be reminded that we still consider functions $f: S \rightarrow G^{*}$, where $(S, \cdot)$ is a semigroup and $\left(G^{*},+\right)$ is a uniquely divisible abelian group.

Theorem $1 f \in \mathcal{A}(S)$ if and only if, for all $x, y \in S$, one of the following properties holds:

(i) $f\left(x y^{n}\right)=f(x)+n(f(x y)-f(x))$ for all $n \in \mathbb{N}$.

(ii) $f\left(x y^{n}\right)=(-1)^{n}(f(x)-n(f(x)+f(x y)))$ for all $n \in \mathbb{N}$.

(iii) $f\left(x y^{n}\right)=-f(x) / 3$ for all $n \in \mathbb{N}$.

(iv) $f\left(x y^{n}\right)=(-1)^{n+1} f(x) / 3$ for all $n \in \mathbb{N}$.

Proof: The sufficiency of the theorem can be directly verified. Thus we will only prove the necessity.

For any $x, y \in S$. There are four possibilities concerning $\mathcal{P}_{f}(x, y)$ and $\mathcal{P}_{f}(x y, y)$ for us to consider.

(i) If $J_{f}^{-}(x, y)=0$ and $J_{f}^{-}(x y, y)=0$, then Corollary 1 gives the first property in the theorem.

(ii) If $J_{f}^{+}(x, y)=0$ and $J_{f}^{+}(x y, y)=0$, then Corollary 2 gives the second property in the theorem. We remark that this case is not exclusive from the first case when $f(x y)=f\left(x y^{2}\right)=0$.

(iii) If $J_{f}^{-}(x, y) \neq 0$ and $J_{f}^{+}(x y, y) \neq 0$, then Lemma 3 gives the third property in the theorem.

(iv) If $J_{f}^{+}(x, y) \neq 0$ and $J_{f}^{-}(x y, y) \neq 0$, then Lemma 4 gives the forth property in the theorem.

We shall demonstrate how one may use Theorem 1 to solve (3) on some specific semigroups. The two following theorems give the results on infinite and finite cyclic semigroups.

Theorem 2 Let $S=\langle a\rangle$ be an infinite cyclic semigroup. Then $f \in \mathcal{A}(S)$ if and only if

(i) $f\left(a^{n}\right)=k_{0}+k_{1} n$ for all $n \in \mathbb{N}$, or 
(ii) $f\left(a^{n}\right)=(-1)^{n}\left(k_{0}+k_{1} n\right)$ for all $n \in \mathbb{N}$, or

(iii) $f\left(a^{n}\right)=\left(1-4 \delta_{n 0}\right) k_{0}$ for all $n \in \mathbb{N}$, or

(iv) $f\left(a^{n}\right)=(-1)^{n}\left(1-4 \delta_{n 0}\right) k_{0}$ for all $n \in \mathbb{N}$, where $k_{0}$ and $k_{1}$ are constants in $G^{*}$, and $\delta_{i j}$ is the Kronecker delta.

Proof: The theorem directly follows from Theorem 1 with $x=y=a$.

Theorem 3 Let $S=\left\langle a \mid a^{m}=a^{m+p}\right\rangle$ be a finite cyclic semigroup with the index $m$ and the period $p$. Then $f \in \mathcal{A}(S)$ if and only if

(i) $f\left(a^{n}\right)=k$ for all $n \in \mathbb{N}$, or

(ii) $f\left(a^{n}\right)=(-1)^{n} k$ for all $n \in \mathbb{N}$ and $2 \mid p$, or

(iii) $f\left(a^{n}\right)=\left(1-4 \delta_{n 0}\right) k$ for all $n \in \mathbb{N}$ if $m>1$, or

(iv) $f\left(a^{n}\right)=(-1)^{n}\left(1-4 \delta_{n 0}\right) k$ for all $n \in \mathbb{N}$ if $m>1$ and $2 \mid p$.

where $k$ is a constant in $G^{*}$, and $\delta_{i j}$ is the Kronecker delta.

Proof: We will identify each possibility in Theorem 3 and apply the conditions of the finite cyclic semigroup.

(i) $f\left(a^{n}\right)=k_{0}+k_{1} n$ for all $n \in \mathbb{N}$. Since $a^{m}=$ $a^{m+p}$, we will have $k_{0}+k_{1} m=k_{0}+k_{1}(m+p)$. Thus $k_{1}=0$ and hence $f\left(a^{n}\right)$ is a constant in $G^{*}$.

(ii) $f\left(a^{n}\right)=(-1)^{n}\left(k_{0}+k_{1} n\right)$ for all $n \in \mathbb{N}$. If $2 \mid p$, then $a^{m}=a^{m+p}$ gives $k_{0}+k_{1} n=k_{0}+k_{1}(m+$ $p$ ), which yields $k_{1}=0$. Thus $f\left(a^{n}\right)=(-1)^{n} k$ for all $n \in \mathbb{N}$, where $k$ is a constant in $G^{*}$.

If $2 \mid p+1$, then $a^{n}=a^{n+p}$ yields $k_{0}+k_{1} n=$ $-\left(k_{0}+k_{1}(n+p)\right)$ for all $n \geqslant m$. Therefore, $k_{0}=k_{1}=0$. Therefore, $f\left(a^{n}\right)=0$ for all $n \in$ $\mathbb{N}$, which has already been taken care of in the first case.

(iii) $f\left(a^{n}\right)=\left(1-4 \delta_{n 0}\right) k_{0}$ for all $n \in \mathbb{N}$. If $m=1$, then $a=a^{1+p}$ gives $-3 k_{0}=k_{0}$. Hence $k_{0}=0$ and this solution has already been taken care of in either of the first two cases.

(iv) $f\left(a^{n}\right)=(-1)^{n}\left(1-4 \delta_{n 0}\right) k_{0}$ for all $n \in \mathbb{N}$. If $m=1$, then $a=a^{1+p}$ implies $-3 k_{0}=$ $(-1)^{1+p} k_{0}$, which gives $k_{0}=0$. If $m>1$ and $2 \mid p+1$, then $a^{m}=a^{m+p}$ implies $k_{0}=-k_{0}$, which again gives $k_{0}=0$. The solution with $k_{0}=0$ has already been taken care of in either of the first two cases.

The next theorem gives the result on a semigroup with 2 idempotent generators.

Theorem 4 Let $H=\left\langle a, b \mid a^{2}=a, b^{2}=b\right\rangle$. Then $f \in \mathcal{A}(H)$ if and only if

(i) $f(a x)=f(a)$ and $f(b x)=f(b)$ for all $x \in H$, or (ii) $f(a x)=-f(a) / 3$ and $f(b x)=f(b)$ for all $x \in$ $H$, or

(iii) $f(a x)=f(a)$ and $f(b x)=-f(b) / 3$ for all $x \in$ $H$, or

(iv) $f(a x)=-f(a) / 3$ and $f(b x)=-f(b) / 3$ for all $x \in H$.

Proof: The sufficiency of the theorem is straightforward. Hence we will only show the necessity. Let $f \in \mathcal{A}(H)$. For any $x \in H$. Considering $\mathcal{P}_{f}(x, a)$ and using $a^{2}=a$, we will have

$$
f(x a) \in\left\{f(x),-\frac{1}{3} f(x)\right\} .
$$

Similarly, considering $\mathcal{P}_{f}(x, b)$ and using $b^{2}=b$ will give

$$
f(x b) \in\left\{f(x),-\frac{1}{3} f(x)\right\} .
$$

For an integer $l>1$ and $x_{1}, x_{2}, \ldots, x_{l} \in\{a, b\}$, we can apply (8) and (9) in a certain order to get

$$
f\left(x_{1} x_{2} \cdots x_{l}\right)=\left(-\frac{1}{3}\right)^{k} f\left(x_{1}\right)
$$

for some integer $k$ with $0 \leqslant k<l$.

Next we will show that

$$
\begin{aligned}
\forall x \in H[f(a x) & =f(a)] \\
& \text { or } \quad \forall x \in H\left[f(a x)=-\frac{1}{3} f(a)\right] .
\end{aligned}
$$

Let $\alpha=f(a)$. If $\alpha=0$, then (10) with $x_{1}=a$ gives $f(a y)=0$, which already satisfies (11). Hence we will assume that $\alpha \neq 0$.

Let $c=a b$. For all $n \in \mathbb{N}$, using (10) with $x_{1}=$ $a$, we obtain

$$
f\left(c^{n}\right)=\left(-\frac{1}{3}\right)^{k} \alpha,
$$

for some integer $k$ with $0 \leqslant k<2 n$ In particular, when $n=1$,

$$
f(c) \in\left\{\alpha,-\frac{1}{3} \alpha\right\} .
$$

Using Theorem 1 with $x=a$ and $y=c$, and observing that $a c^{n}=c^{n}$ for all $n \in \mathbb{N}$. we have 4 possible cases to consider.

(i) $f\left(c^{n}\right)=f(a)+n(f(c)-f(a))$ for all $n \in \mathbb{N}$. If $f(c)=-\alpha / 3$, then $f\left(c^{2}\right)=-5 \alpha / 3$, which is impossible when compared to (12) with $n=2$. Therefore, $f(c)=\alpha$ and consequently $f\left(c^{n}\right)=$ $\alpha=f(c)$ for all $n \in \mathbb{N}$. 
(ii) $f\left(c^{n}\right)=(-1)^{n}(f(a)-n(f(c)+f(a)))$ for all $n \in \mathbb{N}$. If $f(c)=\alpha$, then $f\left(c^{2}\right)=-3 \alpha$, which is impossible when compared to (12) with $n=2$. If $f(c)=-\alpha / 3$, then $f\left(c^{4}\right)=-5 \alpha / 3$. which is impossible when compared to (12) with $n=4$.

(iii) $f\left(c^{n}\right)=-f(a) / 3=-\alpha / 3$ for all $n \in \mathbb{N}$. That is $f\left(c^{n}\right)=f(c)$ for all $n \in \mathbb{N}$.

(iv) $f\left(c^{n}\right)=(-1)^{n+1} f(a) / 3=(-1)^{n+1} \alpha / 3$ for all $n \in \mathbb{N}$. Hence $f(c)=\alpha / 3$, which is impossible when compared to (13).

From all cases considered above, we conclude that

$$
f\left(c^{n}\right)=f(c) \quad \text { for all } n \in \mathbb{N} .
$$

For each $n \in \mathbb{N}$, we will evaluate $f\left(c^{n} a\right)$ in two different ways. First, applying (8) with $x=c^{n}$ and noting from (14) that $f\left(c^{n}\right)=f(c)$, we will get

$$
f\left(c^{n} a\right) \in\{f(c),-f(c) / 3\} .
$$

On the other hand, if we apply (9) with $x=c^{n} a$, we will have $f\left(c^{n} a b\right) \in\left\{f\left(c^{n} a\right),-\frac{1}{3} f\left(c^{n} a\right)\right\}$. But $c^{n} a b=c^{n+1}$ and, from (14), $f\left(c^{n+1}\right)=f(c)$. Therefore,

$$
f\left(c^{n} a\right) \in\{f(c),-3 f(c)\} .
$$

Comparing (15) and (16), we conclude that

$$
f\left(c^{n} a\right)=f(c) \quad \text { for all } n \in \mathbb{N} .
$$

For each $x \in H, a x=c^{n}$ or $a x=c^{n} a$ for some $n \in \mathbb{N}$. Hence from (13), (14) and (17), we have established (11).

A similar result applies to $f(b x)$ where $x \in H$. Hence all possible forms of $f$ in the theorem have been obtained and the proof is now complete.

\section{FURTHER RESULTS ON GROUPS}

In this section, we will investigate the possibilities when the semigroup $S$ possesses additional properties. The next lemma gives a variant of Theorem 1 when the semigroup $(S, \cdot)$ is replaced by a group $(G, \cdot)$. As with previous sections, we let $\left(G^{*},+\right)$ be a uniquely divisible abelian group.

Lemma 5 If $(G, \cdot)$ is a group, then $f \in \mathcal{A}(G)$ if and only if $f$ has one of the following properties.

(i) $f\left(x y^{n}\right)=f(x)+n(f(x y)-f(x))$ for all $x, y \in$ $G, n \in \mathbb{N}$.

(ii) $f\left(x y^{n}\right)=(-1)^{n}(f(x)-n(f(x)+f(x y)))$ for all $x, y \in G, n \in \mathbb{N}$.

Proof: Let $x, y \in G$ and let $f(x)=-3 k$. Suppose that one of the last two properties in Theorem 1 holds. If $f\left(x y^{n}\right)=-f(x) / 3=k$ for all $n \in$ $\mathbb{N}$. Considering $\mathcal{P}_{f}\left(x y^{-1}, y\right)$, we get $f\left(x y^{-1}\right) \in$ $\{5 k,-7 k\}$. Now $\mathcal{P}_{f}\left(x y^{-1}, y^{2}\right)$ implies $k=0$. If $f\left(x y^{n}\right)=(-1)^{n+1} f(x) / 3=(-1)^{n} k$ for all $n \in$ $\mathbb{N}$. Considering $\mathcal{P}_{f}\left(x y^{-1}, y\right)$, we get $f\left(x y^{-1}\right) \in$ $\{-5 k, 7 k\}$. Now $\mathcal{P}_{f}\left(x y^{-1}, y^{2}\right)$ implies $k=0$. We now conclude that $k=0$. Therefore, $f(x)=0$ and $f\left(x y^{n}\right)=0$ for all $n \in \mathbb{N}$, which has already been taken care of in either of the first two properties in Theorem 1. Therefore, the last two properties in Theorem 1 are unnecessary for functions defined on the group $(G, \cdot)$.

The next theorem will extend Lemma 5 to account for $f\left(x y^{n}\right)$ when $n$ is a negative integer.

Theorem 5 If $(G, \cdot)$ is a group, then $f \in \mathcal{A}(G)$ if and only if $f$ has one of the following properties.

(i) $f\left(x y^{n}\right)=f(x)+n(f(x y)-f(x))$ for all $x, y \in$ $G, n \in \mathbb{Z}$.

(ii) $f\left(x y^{n}\right)=(-1)^{n}(f(x)-n(f(x)+f(x y)))$ for all $x, y \in G, n \in \mathbb{Z}$.

Proof: The sufficiency of the theorem is obvious. To prove the necessity, we let $f \in \mathcal{A}(G)$ and let $x, y \in G$. From Lemma 5, there are two possibilities.

(i) Consider the first possibility when

$$
f\left(x y^{n}\right)=f(x)+n(f(x y)-f(x))
$$

for all $n \in \mathbb{N}$. Then setting $n=2$ and $n=3$, we get

$$
\begin{aligned}
& f\left(x y^{2}\right)=2 f(x y)-f(x), \\
& f\left(x y^{3}\right)=3 f(x y)-2 f(x) .
\end{aligned}
$$

Replacing $x$ and $y$ in Lemma 5 with $x y^{3}$ and $y^{-1}$, respectively, we have two more possibilities to explore.

(a) If $f\left(x y^{3-n}\right)=f\left(x y^{3}\right)+n\left(f\left(x y^{2}\right)-f\left(x y^{3}\right)\right)$ for all $n \in \mathbb{N}$, then substituting $f\left(x y^{2}\right)$ and $f\left(x y^{3}\right)$ from (19) and (20), we get $f\left(x y^{3-n}\right)=f(x)+(3-n)(f(x y)-f(x))$ for all $n \in \mathbb{N}$. Hence (18) holds for all $n \in \mathbb{Z}$

(b) If $f\left(x y^{3-n}\right)=(-1)^{n}\left(f\left(x y^{3}\right)-n\left(f\left(x y^{3}\right)+\right.\right.$ $\left.\left.f\left(x y^{2}\right)\right)\right)$ for all $n \in \mathbb{N}$. then setting $n=2$ and $n=3$, we get

$$
\begin{gathered}
f(x y)=-2 f\left(x y^{2}\right)-f\left(x y^{3}\right) . \\
f(x)=3 f\left(x y^{2}\right)+2 f\left(x y^{3}\right),
\end{gathered}
$$

From (19), (20), (21) and (22), we have $f\left(x y^{3}\right)=f\left(x y^{2}\right)=f(x y)=f(x)=0$. Hence $f\left(x y^{n}\right)=0$ for all $n \in \mathbb{Z}$, and so (18) holds for all $n \in \mathbb{Z}$. 
(ii) We turn to the second possibility when

$$
f\left(x y^{n}\right)=(-1)^{n}(f(x)-n(f(x)+f(x y)))
$$

for all $n \in \mathbb{N}$. Then setting $n=2$ and $n=3$, we get

$$
\begin{aligned}
& f\left(x y^{2}\right)=-2 f(x y)-f(x), \\
& f\left(x y^{3}\right)=3 f(x y)+2 f(x) .
\end{aligned}
$$

Replacing $x$ and $y$ in Lemma 5 with $x y^{3}$ and $y^{-1}$, respectively, we have two more possibilities to explore.

(a) If $f\left(x y^{3-n}\right)=f\left(x y^{3}\right)+n\left(f\left(x y^{2}\right)-f\left(x y^{3}\right)\right)$ for all $n \in \mathbb{N}$. Then setting $n=2$ and $n=3$ gives

$$
\begin{aligned}
f(x y) & =2 f\left(x y^{2}\right)-f\left(x y^{3}\right) . \\
f(x) & =3 f\left(x y^{2}\right)-2 f\left(x y^{3}\right),
\end{aligned}
$$

From (24), (25), (26) and (27), we have $f\left(x y^{3}\right)=f\left(x y^{2}\right)=f(x y)=f(x)=0$. Hence $f\left(x y^{n}\right)=0$ for all $n \in \mathbb{Z}$ and (23) holds for all $n \in \mathbb{Z}$.

(b) If $f\left(x y^{3-n}\right)=(-1)^{n}\left(f\left(x y^{3}\right)-n\left(f\left(x y^{3}\right)+\right.\right.$ $\left.\left.f\left(x y^{2}\right)\right)\right)$, then substituting $f\left(x y^{2}\right)$ and $f\left(x y^{3}\right)$ from (24) and (25), we get $f\left(x y^{3-n}\right)=(-1)^{n}(f(x)-(3-n)(f(x)+$ $f(x y)))$ for all $n \in \mathbb{N}$. Hence (23) holds for all $n \in \mathbb{Z}$

We will now take a step further by assuming that the group $(G, \cdot)$ is 2-divisible; that is, for all $x \in G$, there exist $y \in G$ such that $x=y^{2}$. The next theorem will establish the equivalence of the alternative Jensen's functional equation (3) and Jensen's functional equation (2) for functions defined on a 2-divisible group.

Theorem 6 If $(G, \cdot)$ is a 2-divisible group, then $f \in$ $\mathcal{A}(G)$ if and only if $f$ satisfies (2) for all $x, y \in G$.

Proof: The sufficiency of the theorem is obvious. To prove the necessity, we let $x, y \in G$. Since $G$ is 2 divisible, there exists an element $z \in G$ such that $y=$ $z^{2}$. Using Theorem 5 with $y$ replaced by $z$, we will prove that $f(x)-2 f\left(x z^{2}\right)+f\left(x z^{4}\right)$ by considering two cases.

(i) If $f\left(x z^{n}\right)=f(x)+n(f(x z)-f(x))$ for all $n \in \mathbb{N}$. With $n=2$ and $n=4$, we have $f\left(x z^{2}\right)=$ $2 f(x z)-f(x)$ and $f\left(x z^{4}\right)=4 f(x z)-3 f(x)$, respectively. Eliminating $f(x z)$ gives $f(x)-$ $2 f\left(x z^{2}\right)+f\left(x z^{4}\right)=0$. (ii) If $f\left(x z^{n}\right)=(-1)^{n}(f(z)-n(f(x z)+f(z)))$ for all $n \in \mathbb{N}$. With $n=2$ and $n=4$, we have $f\left(x z^{2}\right)=-2 f(x z)-f(x)$ and $f\left(x z^{4}\right)=$ $-4 f(x z)-3 f(x)$, respectively. Eliminating $f(x z)$ gives $f(x)-2 f\left(x z^{2}\right)+f\left(x z^{4}\right)=0$.

Recalling that $y=z^{2}$, we conclude from both cases above that $f(x)-2 f(x y)+f\left(x y^{2}\right)=0$. Hence $f$ satisfies (2) for all $x, y \in G$.

\section{REFERENCES}

1. Kuczma M (1978) On some alternative functional equations. Aequationes Math 17, 182-98.

2. Kuczma M (1978) Functional equations on restricted domains. Aequationes Math 18, 1-34.

3. Forti GL (1979) La soluzione generale dell'equazione funzionale $\{c f(x+y)-a f(x)-b f(y)-d\}\{f(x+y)-$ $f(x)-f(y)\}=0$. Matematiche (Catania) 34, 219-42.

4. Ger R (1976) On a method of solving of conditional Cauchy equations. Univ Beograd Publ Elektrotechn Fak Ser Mat Fiz, 544-576, 159-65.

5. Forti GL, Paganoni L (1981) A method of solving of a conditional Cauchy equation on abelian groups. Ann Mat Pura Appl 127, 77-99.

6. Paganoni L, Rätz J (1995) Conditional functional equation and orthogonal additivity. Aequationes Math 50, 135-42.

7. Ng CT (1990) Jensen's functional equation on groups. Aequationes Math 39, 85-99.

8. Parnami JC (1992) On Jensen's functional equation. Aequationes Math 43, 211-8. 